\title{
Submandibular Abscess with Velopharyngeal Insufficiency: Unusual Clinical Presentation of Tuberculosis
}

\author{
Swati Tandon*, Purodha Prasad, Vikram Wadhwa and Ishwar Singh \\ Maulana Azad Medical College, India
}

Submission: February 20, 2017; Published: June 16, 2017

*Corresponding author: Swati Tandon, Maulana Azad Medical College, Delhi, India, Tel: 9891778593; Email: drswatitandon86@gmail.com

Abstract

Tuberculosis is a major public health problem in India. The rising incidence of multi drug resistant tuberculosis and unusual presentations of the disease is posing a great challenge for clinicians. We report an interesting case of 27 year old male who initially presented with submandibular abscess, subsequently developed velopharyngeal insufficiency during hospital stay and finally diagnosed as a case of extrapulmonary tuberculosis.

\section{Introduction}

Tuberculosis is one of the oldest diseases known to affect humans. There are two forms of tuberculosis: pulmonary and extrapulmonary. Extrapulmonary tuberculosis involves all sites other than lungs. Diagnosis of extrapulmonary tuberculosis is challenging as samples obtained from these sites may be paucibacillary, thus decreasing the sensitivity of diagnostic tests [1]. We report a case of unusual presentation of disseminated extrapulmonary tuberculosis, where patient initially presented as an acute submandibular abscess, then developed palatal perforation and later had massive pleural effusion. Pleural fluid analysis ultimately leads to the diagnosis of tuberculosis.

\section{Case Report}

A 27 year old male presented to ENT emergency of our hospital with diffuse swelling below the chin for 6 days. The swelling initially started as a furuncle which gradually progressed to involve the whole region below the chin. It was associated with pain and difficulty in eating. There was no breathing difficulty or fever. There was no history of trauma. There was no history of contact or past tuberculosis. On clinical examination, patient was thin built, cachexic and a febrile.

On local examination of neck, a diffuse $8 \times 8 \mathrm{~cm}$ swelling was seen in neck below the mandible extending from one angle of mandible to other. It was tender, skin overlying was erythematous and hyper pigmented, and temperature was raised. It was fluctuant and on aspiration pus was aspirated. A provisional diagnosis of acute submandibular abscess was made and incision and drainage was done with $10 \mathrm{ml}$ drainage of pus. Floor of mouth was raised which subsided on drainage of abscess. Pus was sent for Gram staining, ZN staining (for TB) and culture sensitivity. Acid fast bacilli (AFB) were negative on ZN staining. On culture, pseudomonas was isolated and appropriate intravenous antibiotics were started according to sensitivity report. Hematological investigations including complete blood count, kidney function tests, liver function tests and urine routine microscopy was done, which were within normal limits. No immunodeficiency was detected. On second day, patient complained of nasal regurgitation and regurgitation of water through right ear. Voice of the patient also appeared hyper nasal. Nasal examination was unremarkable. On oropharyngeal examination, approx. $1 \mathrm{~cm}$ ulcer with perforation was seen at the junction of the right anterior pillar and soft palate with pus on its margins. Patient denied any history of trauma or drug allergy. On examination of right ear, pus was seen filling the external auditory canal with non visualization of tympanic membrane. On cleaning the pus, slough was seen in anteroinferior wall of external auditory canal in the cartilaginous portion with pus coming through it. Tympanic membrane was found intact. Pus in the ear was thought to be due to spread of infection via the parotid space into the external auditory canal (EAC) via fissure of Santorini. Approx. 10-15ml pus drained from the submandibular incision site on second day. Patient was continued on i.v. antibiotics. 
Nasogastric tube was inserted for feeding and biopsy was taken from the ulcer margins which showed chronic inflammation without any granulomas and was negative for acid fast bacilli. On third day, patient started complaining of purulent cough with mild respiratory difficulty. Chest physician opinion was sought who ordered chest $\mathrm{x}$ ray, montoux, sputum for AFB smear and started patient on tab. levofloxacin for 7 days. On chest $\mathrm{X}$ ray, normal lung parenchyma with blunting of bilateral $\mathrm{CP}$ angles were seen indicating pleural effusion for which ultrasound guided pleural tap was done which revealed $4 \mathrm{~cm}$ pleural thickness on right side and $5 \mathrm{~cm}$ on left side. Montoux was $10 \mathrm{~mm}$, sputum for AFB was negative and ESR was raised $(72 \mathrm{~mm} / \mathrm{hr})$.

Analysis of pleural fluid revealed raised lymphocytic count raised LDH and ADA s/o tuberculosis. Thus, a diagnosis of extrapulmonary tuberculosis was made and patient was started on ATT. Initially, patient did not respond and respiratory distress worsened. Repeat chest $\mathrm{x}$ ray revealed massive pleural effusion for which chest drains were inserted bilaterally. $1000 \mathrm{ml}$ pus was drained from right side and $450 \mathrm{ml}$ from left side. Gradually patient started improving.

Chest drains were removed after 3 days. Submandibular wound also started healing and healthy granulation tissue was formed on 10th day. Anterior pillar perforation also healed by 10 th day (Figure $1 \& 2$ ). Patient's general condition improved and was discharged after 2 weeks on ATT. On follow up of 8 weeks, patient is doing well and neck wound has healed.

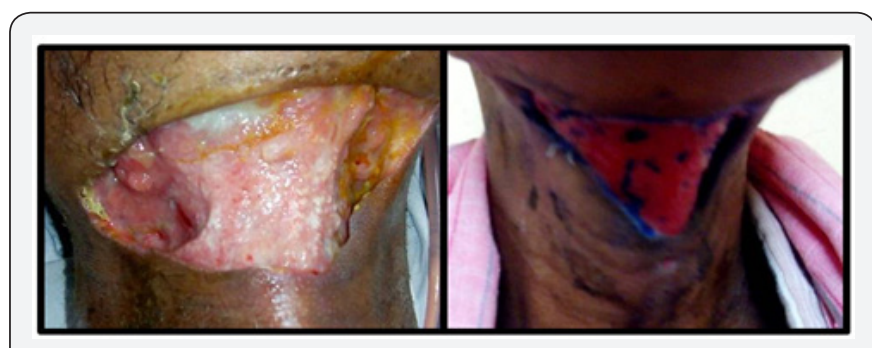

Figure 1: Pre and Post Treatment Photograph of Submandibular Abscess.

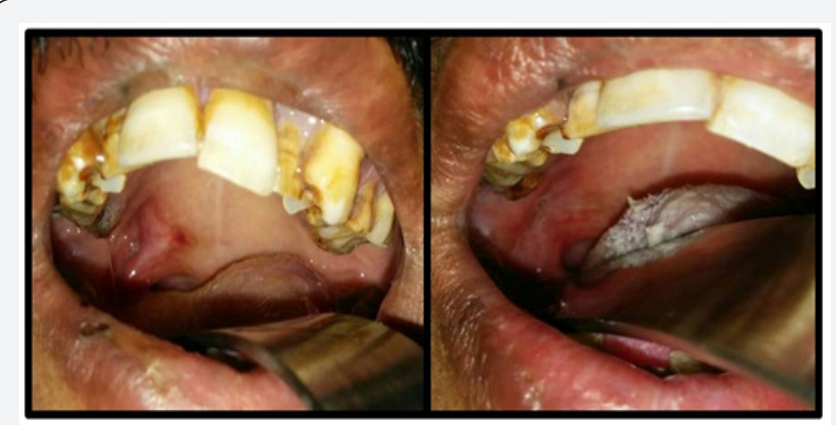

Figure 2: Pre and Post Treatment Photograph of Palatal Perforation.

\section{Discussion}

With the advent of HIV infection, extrapulmonary tuberculosis (EPTB) is being increasingly reported [2]. Other risk factors predisposing to EPTB are chronic renal failure, diabetes, immunosuppressive treatment, intravenous drug abuse, post organ transplantation and severe malnutrition. Diagnosis of EPTB is based on one culture-positive specimen from the extrapulmonary site; or histological evidence; or strong clinical evidence consistent with active EPTB disease followed by treatment with a full course of anti-TB therapy [3]. In India, EPTB forms 10 to 15 percent of all types of TB [3].

The major pitfalls in the diagnosis of EPTB are atypical clinical presentations simulating other inflammatory conditions, resulting in delay of treatment. Therefore, a high index of suspicion is necessary to make an early diagnosis. In developing countries, the lack of diagnostic resources adds to the problems. In clinical practice, the cutaneous reaction to PPD commonly known as montoux test is used as an aid to diagnose TB but its value as a diagnostic tool is limited in adults in India, since about $40 \%$ of the adult population is infected with TB. In our case, montoux was $10 \mathrm{~mm}$ suggestive of TB.

Smear examination for AFB, culture and histopathological examination remain as the classical diagnostic tests for TB. Laboratory diagnosis of $\mathrm{TB}$ is a tedious process because it depends on the growth of organisms. ZN staining for demonstration of acid fast bacilli on smears is a rapid method but it is less sensitive. In a study, ZN staining was compared with fluorescent (auramine rhodamine-AR) staining for demonstration of acid fast bacilli and it was observed that ZN staining showed $23.4 \%$ AFB smear positivity; $32.7 \%$ in sputum and $1.4 \%$ in extra-pulmonary specimens, whereas, Auramine Rhodamine staining showed $31.87 \%$ AFB smear positivity, $41.6 \%$ in sputa and $9.9 \%$ in extrapulmonary cases. The staining methods were also compared and evaluated against culture on LJ medium, (taken as 'gold standard'): AR was $86.6 \%$ sensitive and ZN $67.3 \%$ sensitive [4].

Culture is the gold standard method but it's major disadvantage is it is time consuming and takes 3 to 8 weeks. The most recent advances have been development of molecular tools for amplifying DNA and RNA in clinical samples. A new nucleic acid amplification test called transcription mediated amplification has been developed [5]. These tests enable rapid identification of bacilli in few hours and are highly sensitive and specific. Also, since they involve amplification of bacilli DNA and RNA, they are very useful in paucibacillary specimens. Histopathological examination for mycobacterial lesions has also been described as a diagnostic tool. It has been found that microscopic examination of tissue sections frequently results in few or no bacilli seen, even if the lesions appear active histologically. This might be due to the effects of the fixative fluid and/or organic solvent, both of which are conventionally used to make tissue sections for histopathology, on the acid-fast staining of bacteria [6]. We also suspect the same in our case.

Measurement of adenosine deaminase (ADA) activity is one of the most widely used biomarkers in body fluids for 
the diagnosis of EPTB. ADA is an enzyme involved in purin metabolism. Activity of this enzyme increases in TB patients because of the stimulation of T-cell lymphocytes by mycobacterial antigens. Detection of ADA in pleural fluid ultimately helped in establishing the diagnosis in our case.

TB of upper airway and oral cavity is usually secondary to pulmonary TB. Cases of primary oral TB have been described in literature [7]. On the other hand oral TB can be the first sign of pulmonary TB [8]. In our case, oral TB was one of the manifestations of extrapulmonary TB. Antituberculosis treatment is the mainstay in the management of EPTB.

\section{Conclusion}

Our case highlights the varied and unusual presentations of extrapulmonary tuberculosis. It also highlights the limitations of diagnostic tests routinely used for diagnosing TB. Extrapulmonary manifestation of TB can affect any part of body; therefore high clinical suspicion is needed to diagnose such cases early so as to prevent complications and spread of infection to others.

\section{References}

1. JY Lee (2015) Diagnosis and Treatment of Extrapulmonary Tuberculosis. Tuberc Respir Dis (Seoul) 78(2): 47-55.

2. Harrison's Principles of Internal Medicine Vol 1. (17 th edn), pp. 1008.

3. Fraser Wares, Balasubramanian $\mathrm{R}$, Mohan A, Sharma SK Extrapulmonary Tuberculosis: Management and Control. Tuberculosis Control in India. Chapter 11. Pp 95.

4. Jain A, Bhargava A, Agarwal SK (2002) A Comparative Study of two commonly used staining techniques for acid fast bacilli in clinical specimens. Ind J Tub 49: 161.

5. Shenai S, Rodrigues C, Almeida A, Mehta A (2001) Rapid diagnosis of tuberculosis using transcription mediated amplification. Indian Journal of Medical Microbiology 19(4): 184-189.

6. Hajime F, Tomoyuki M, Toshikazu G, Kazuo S, Tokuhiro I (2002) Sensitivity of Acid-Fast Staining for Mycobacterium tuberculosis in Formalin-fixed Tissue. Am J Respir Crit Care Medicine 166(7): 994997.

7. Choi KW, Kim YH, Chung KJ (2015) Experience of Adult-Onset Velopharyngeal Insufficiency after Tuberculosis of the Soft Palate. Arch Plast Surg 42(1): 89-91.

8. Sharma N, Malhotra D, Kaur J (2013) Tubercular Palatal Perforation: First Clinical Sign of Undiagnosed Pulmonary Tuberculosis. Indian Journal of Applied Research 8(3): 74-5.

\section{Your next submission with Juniper Publishers} will reach you the below assets

- Quality Editorial service

- Swift Peer Review

- Reprints availability

- E-prints Service

- Manuscript Podcast for convenient understanding

- Global attainment for your research

- Manuscript accessibility in different formats

( Pdf, E-pub, Full Text, Audio)

- Unceasing customer service

Track the below URL for one-step submission https://juniperpublishers.com/online-submission.php 